\title{
A touchy subject: an assessment of cutaneous allodynia in a chronic migraine population
}

This article was published in the following Dove Press journal:

Journal of Pain Research

24 February 2016

Number of times this article has been viewed

\section{Paul G Mathew ${ }^{1-4}$ \\ Fred Michael Cutrer ${ }^{5}$ \\ Ivan Garza ${ }^{5}$}

'Department of Neurology, John R Graham Headache Center, Brigham and Women's Hospital, Boston, MA, ${ }^{2}$ Department of Neurology, Harvard Vanguard Medical Associates, Braintree, MA, ${ }^{3}$ Division of Neurology, Cambridge Health Alliance, Cambridge, MA, ${ }^{4}$ Harvard Medical School, Boston, MA, ${ }^{5}$ Department of Neurology, Mayo Clinic, Rochester, MN, USA
Correspondence: Paul G Mathew Department of Neurology, John R Graham Headache Center, Brigham and Women's Hospital, I I 53 Centre Street, Suite 4970, Boston, MA 02130, USA

$\mathrm{Tel}+\mathrm{I} 6179837580$

Fax + I 6179837582

Email pmathew@partners.org
Background: Cutaneous allodynia (CA) is a common feature of migraine, which has a complex underlying pathophysiology that is not well understood. In addition to pain, photophobia, phonophobia, osmophobia, nausea, and vomiting, CA can contribute to the overall disability caused by migraine. The presence of CA can be established via a validated questionnaire. Validated questionnaires and other tests are rarely performed in clinical practice. As such, current prevalence estimates for CA may be an underestimation.

Methods: Utilizing a validated questionnaire, we assessed the presence of CA in consecutive patients $(n=44)$ presenting with chronic migraine at a tertiary headache center.

Results: CA appears to be quite prevalent, at $\sim 90 \%$, among female patients with chronic migraine.

Conclusion: CA prevalence in chronic migraine may be underestimated in the literature, and larger studies may better demonstrate a more accurate estimate of its prevalence.

Keywords: headache, validated questionnaire, migraine chronification, central sensitization

\section{Background}

Migraine is one of the most common headache disorders for which patients present to a physician for evaluation, with a prevalence of $18 \%$ in women and $6 \%$ in men. ${ }^{1,2}$ Migraine is a primary headache disorder that probably occurs as a result of one or more variants in the genetic code. ${ }^{3}$ The exact mechanisms by which susceptibility is related to initiation of migraine attacks are not completely understood.

Cortical spreading depression (CSD) is a wave of neuronal hyperexcitation followed by suppression that crosses neurovascular territories, and is thought to be the inciting event that triggers a migraine. CSD is also thought to be the cause of aura, and the gradual spread of CSD correlates with the gradual expanding nature of visual and sensory aura. ${ }^{4}$ CSD leads to vascular changes, release of inflammatory neuropeptides, and subsequent central sensitization. ${ }^{5,6}$

In addition to this cascade of excitatory events, in migraineurs, there may also be dysfunction of the descending central nervous system pathways, which normally function to inhibit nociceptive transmission. ${ }^{7}$ The periaqueductal gray, nucleus cuneiformis, and rostral ventral medulla have all been implicated to varying degrees as being involved in descending modulation of nociceptive transmission. ${ }^{8}$

In rat models, it was found that inducing a single, high-intensity meningeal nociceptive stimulation with an injectable inflammatory soup caused reversible cutaneous hypersensitivity. ${ }^{9}$ Similar reversible cutaneous hypersensitivity was generated 
with repetitive, but not single, low-intensity stimulation. Fos expression, which can be a marker for neuronal activity, increased after a single high-intensity stimulation. Repeated high-intensity stimulation led to increase in neuronal hyperexcitability, impairment of inhibitory pathways, increase in trigeminal central sensitization, and persistent cutaneous hypersensitivity. ${ }^{9}$

Central sensitization is thought to result in many features of migraine, including throbbing quality, photophobia, phonophobia, osmophobia, nausea, and vomiting. In addition to these features, many patients experience cutaneous allodynia (CA), which refers to nonnoxious skin stimulation causing an unpleasant or painful response. As such, the presence of CA can be a useful marker for central sensitization.

In rat models, the application of inflammatory soup on the dura with coadministration of sumatriptan effectively blocked the development of CA as the neuronal response threshold to skin stimulation did not decrease. In contrast, a 2-hour delay in sumatriptan administration caused the neuronal response to skin brushing to remain elevated after the development of $\mathrm{CA} .^{10}$

$\mathrm{CA}$ is not a part of the International Classification of Headache Disorders, Third Edition, Beta Version (ICHD-III Beta) criteria for migraine, and as such, the presence of CA is often not routinely assessed in clinical practice. ${ }^{11}$ Although currently not a part of the diagnostic criteria for migraine, the presence of CA may serve as a predictor of migraine chronification.

Prevalence estimates of CA have been variable across different populations. In a study conducted in United States, 44 subjects with episodic migraine were tested for mechanical and thermal pain thresholds in the absence of and during a migraine. Among the subjects, 79\% were found to have CA. ${ }^{12}$

In another US study, 89 subjects with chronic migraine were tested for brush allodynia. Thirty-five patients were tested for allodynia while they were having an acute headache exacerbation and 54 were tested when they had their baseline headache. In total, 38 patients (43\%) exhibited brush allodynia. ${ }^{13}$

In a web based survey conducted in the Netherlands involving 2,331 migraineurs, 1,624 (70\%) were found to have CA. Lifetime depression, female sex, low age of onset, and high migraine frequency were all risk factors for CA. In addition, CA was an independent predictor for increase in migraine frequency over a mean follow-up period of 93 \pm 30 weeks (range: $26-160$ weeks). ${ }^{14}$ On the basis of this study, CA appears to be a risk factor for migraine chronification, and detecting the presence of CA may influence therapeutic decision making in terms of abortive and preventive treatment strategies. The gold standard for identification of CA is quantitative sensory testing (QST) performed both between and during attacks. This method of identification is time consuming and impractical for routine clinical use.

A validated questionnaire is a tool that can be utilized to determine the presence of CA. A 12-item questionnaire was devised to assess skin sensitivity during a migraine. This questionnaire has previously been validated by determining pain thresholds to mechanical and thermal stimulation using QST in the absence and presence of a migraine. ${ }^{15}$

The objective of this study was to estimate the prevalence of CA in a US population of subjects with chronic migraine at a tertiary headache center using a validated questionnaire.

\section{Methods}

This study was conducted at the Mayo Clinic, Rochester, MN. It was approved by the Investigational Review Board of the Mayo Clinic.

Consecutive patients diagnosed by a headache neurologist with chronic migraine were invited to participate in the study. Study neurologists assigned a headache diagnosis according to ICHD-III Beta criteria. Patients who provided written informed consent to participate were enrolled in the study. Subjects provided baseline data for the study at the enrollment visit.

Information was collected from all subjects at enrollment on demographic and headache characteristics, including age, sex, age of onset of migraines, and presence of aura. Subjects were classified as allodynic or not if they endorsed one or more items on a 12-item questionnaire designed to identify allodynia during a migraine. ${ }^{15}$ The questionnaire included 12 questions. It assessed whether combing hair, placing hair into a ponytail, shaving, wearing eyeglasses, wearing contact lenses, wearing earrings, wearing necklaces, wearing tight clothes, taking a shower with water hitting the face, resting the face on a pillow on the side of the headache, heat exposure, and cold exposure were painful or unpleasant during a migraine. ${ }^{15}$

Table I Demographic and clinical characteristics of subjects

\begin{tabular}{ll}
\hline & Subjects, $\mathrm{n}=\mathbf{4 4}$ \\
\hline Average age, years (range) & $39.3(18-63)$ \\
Subjects with aura & 17 \\
Mean age of migraine onset & 19 \\
\hline
\end{tabular}




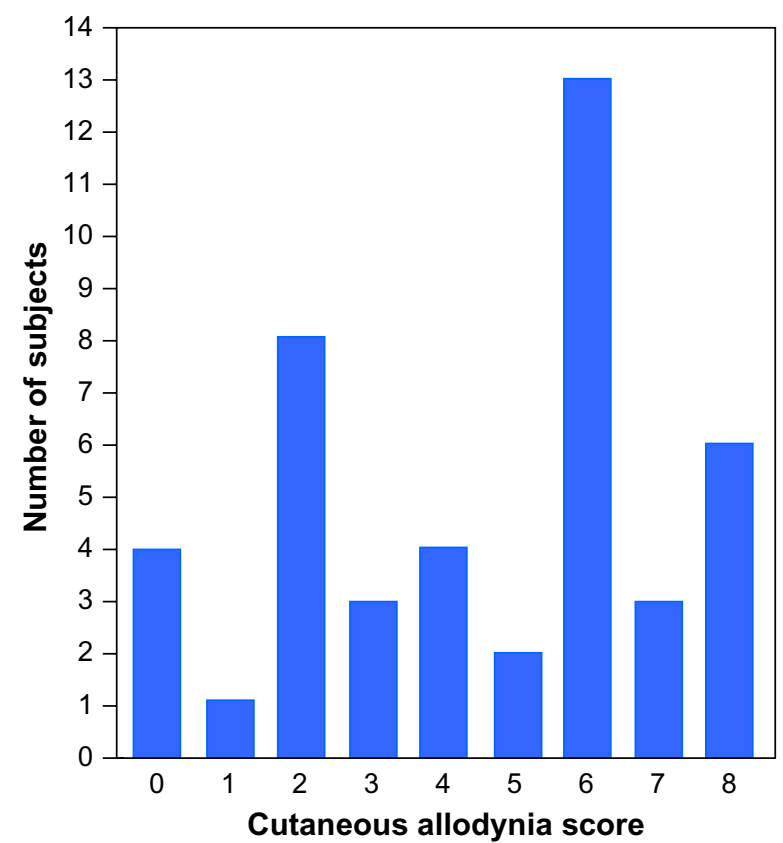

Figure I Breakdown of allodynia scores.

\section{Results}

The baseline characteristics of the subjects are listed in Table 1. The breakdown of allodynia scores is presented in Figure 1. Only one subject chose not to continue in the study after enrolling, for personal reasons. Among 44 subjects, 40 were found to have $\mathrm{CA}$ based on their validated questionnaire score.

\section{Discussion}

$\mathrm{CA}$ is a common feature of migraine and can be considered a clinical marker for central sensitization. As expected, migraineurs with CA have been noted to have lower thermal pain thresholds on cutaneous testing during a migraine. Although this lower thermal pain threshold tends to improve between attacks, both episodic and chronic migraineurs tend to have a lower thermal pain threshold interictally 48 hours after their previous attack than nonmigraine controls. This interictal lower thermal pain threshold may increase the tendency to develop a migraine and may serve as an index of overall migraine activity. ${ }^{10}$

In a functional magnetic resonance imaging (MRI) study using blood oxygen-level-dependent (BOLD) contrastsensitive sequences, allodynic migraineurs tended to have more prominent periaqueductal gray and nucleus cuneiformis resting state connectivity with other brainstem, thalamic, insula, and cerebellar regions that process pain when compared to migraineurs without prominent allodynia. Resting state connectivity in allodynic migraineurs also tended to be stronger, with frontal and temporal regions suggesting differences in higher-order pain modulation. ${ }^{16}$

These thermal threshold and MRI BOLD imaging data further suggest that the presence of CA is probably a risk factor for the transformation from episodic to chronic migraine, and detecting the presence of CA may influence therapeutic decision making in terms of abortive and preventive treatment strategies. On the basis of our data, CA appears to be quite prevalent, at $\sim 90 \%$, in this chronic migraine US population. This may be in part due to the high potential sensitivity of the questionnaire utilized because a subject who could recall a single attack during which he/she found one of the items assessed as unpleasant would be labeled as having CA.

The lower prevalence estimates of CA in the literature may be in part due to the percentage of subjects having episodic migraine, the quality/intensity of sensory stimulation used to trigger a response from the subject, as well as the variability among study populations that were involved. Larger studies may better demonstrate a more accurate estimate of this prevalence. Future CA studies may also help better elucidate the ascending and descending central nervous system pathways, as well as the sequence of pathologic events that culminate in the form of central sensitization and the disabling features of migraine.

A weakness of our study was that it was conducted at a tertiary headache center, and the consecutive migraine subjects who were enrolled all happened to be women, which limits generalizability. In addition, comorbidity data were not collected, which may have provided insight into what other conditions may predispose to CA in a chronic migraine population. The authors were unaware of Allodynia Symptom Checklist (ASC-12) scoring system ${ }^{17}$ at the time this study was conducted, which could have been used instead of the validated CA questionnaire. It is, however, unlikely that use of the ASC-12 would have altered the final results of this study.

\section{Clinical implications}

- The prevalence of CA among subjects with chronic migraine in this study was $\sim 90 \%$, which is higher than the estimates for episodic and mixed episodic/chronic migraine populations.

- CA is probably a risk factor for the chronification from episodic to chronic migraine.

- CA is a marker for central sensitization, which has clinical implications in terms of timing of abortive therapy administration and CA onset. 


\section{Acknowledgment}

This study received no outside funding.

\section{Disclosure}

The authors report no conflicts of interest in this work.

\section{References}

1. Lipton RB, Stewart WF, Simon D. Medical consultation for migraine: results from the American Migraine Study. Headache. 1998;38:87-96.

2. Lipton RB, Stewart WF, Diamond S, Diamond ML, Reed M. Prevalence and burden of migraine in the United States: data from the American Migraine Study II. Headache. 2001;41:646-657.

3. Zhao H, Eising E, de Vries B, et al; International Headache Genetics Consortium. Gene-based pleiotropy across migraine with aura and migraine without aura patient groups. Cephalalgia. Epub 2015 Dec 8.

4. Milner P. Note on a possible correspondence between the scotomas of migraine and spreading depression of Leao. Electroencephalogr Clin Neurophysiol. 1958;10:705.

5. Goadsby PH, Edvinsson L, Ekman R. Vasoactive peptide release in the extracerebral circulation of humans during migraine headache. Ann Neurol. 1990;28:183-187.

6. Yamamura H, Makick A, Chamberlin NL, Burstein R. Cardiovascular and neuronal responses to head stimulation reflect central sensitization and cutaneous allodynia in a rat model of migraine. $J$ Neurophysiol. 1999;81:479-493.

7. Bingel U, Tracey I. Imaging CNS modulation of pain in humans. Physiology (Bethesda). 2008;23:371-380.
8. Schwedt TJ, Larson-Prior L, Coalson RS, et al. Allodynia and descending pain modulation in migraine: a resting state functional connectivity analysis. Pain Med. 2014;15(1):154-165.

9. Boyer N, Dallel R, Artola A, Monconduit L. General trigeminospinal central sensitization and impaired descending pain inhibitory controls contribute to migraine progression. Pain. 2014;155(7):1196-1205.

10. Burstein R, Jakubowski M. Analgesic triptan action in an animal model of intracranial pain: a race against the development of central sensitization. Ann Neurol. 2004;55(1):27-36.

11. Headache Classification Committee of the International Headache Society (IHS). The international classification of headache disorders, 3rd edition (beta version). Cephalalgia. 2013;33(9):629-808.

12. Burstein R, Yarnitsky D, Goor-Aryeh I, Ransil BJ, Bajwa ZH. An association between migraine and cutaneous allodynia. Ann Neurol. 2000;47(5):614-624.

13. Ashkenazi A, Sholtzow M, Shaw JW, Burstein R, Young WB. Identifying cutaneous allodynia in chronic migraine using a practical clinical method. Cephalalgia. 2007;27(2):111-117.

14. Louter MA, Bosker JE, van Oosterhout WP, et al. Cutaneous allodynia as a predictor of migraine chronification. Brain. 2013; 136(pt 11):3489-3496.

15. Jakubowski M, Silberstein S, Ashkenazi A, Burstein R. Can allodynic migraine patients be identified interictally using a questionnaire? Neurology. 2005;65:1419-1422.

16. Schwedt TJ, Krauss MJ, Frey K, Gereau RW IV. Episodic and chronic migraineurs are hypersensitive to thermal stimuli between migraine attacks. Cephalalgia. 2011;31(1):6-12.

17. Lipton RB, Bigal ME, Ashina S, et al. Cutaneous allodynia in the migraine population. Ann Neurol. 2008;63(2):148-158.
Journal of Pain Research

\section{Publish your work in this journal}

The Journal of Pain Research is an international, peer-reviewed, open access, online journal that welcomes laboratory and clinical findings in the fields of pain research and the prevention and management of pain. Original research, reviews, symposium reports, hypothesis formation and commentaries are all considered for publication.

\section{Dovepress}

The manuscript management system is completely online and includes a very quick and fair peer-review system, which is all easy to use. Visit http://www.dovepress.com/testimonials.php to read real quotes from published authors. 